\title{
Frank Ramsey, una estrella fugaz en el universo matemático
}

| Frank Ramsey, a shooting star in the mathematical universe |

\author{
Vernor Arguedas T. \\ vernor.arguedas@ucr.ac.cr \\ Escuela de matemática \\ Universidad de Costa Rica, Costa Rica
}

Recibido: 18 agosto 2020

Aceptado: 28 agosto 2020

Resumen. Se presentan diversos aspectos de la corta pero brillante vida de Frank Ramsey, cuyos logros siguen marcando pautas.

Palabras clave: Ramsey, Keynes, Wittgenstein, verdad, lógica de tipos.

Abstract. Various aspects of the short but brilliant life of Frank Ramsey are presented, whose achievements continue to set the path.

KeyWords: Ramsey, Keynes, Wittgenstein, true, type logic.

\subsection{Breve biografía}

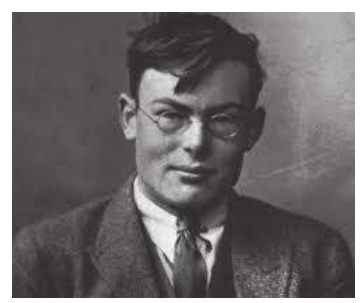

El 22 de febrero de 1903 nació Frank Plumptom Ramsey en el seno de una familiaacomodada e intelectual de Cambridge, Inglaterra, bajo el reinado de Eduardo VII, hijo de Agnes Mary Wilson y Lord Arthur Stanley Ramsey, matemático de la universidad de Cambridge y director del Magdelene College. Ramsey tuvo dos hermanas Bridget (1908) y Margararet (1918) y un hermano, Michael Ramsey, quien fue arzobispo de Canterbury entre 1961 y 1974. Frank nació amarillo con ictericia.

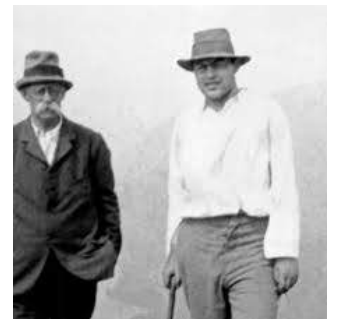

Su madre fue una luchadora social, sufragista y defensora de los derechos de las mujeres, su hermana Margaret fue una destacada escritora autora de una entrañable biografía de su hermano Frank: "Margaret Paul, Frank Ramsey (1903-1930) A sister's memoir" en la cual de manera cronológica presentó diversos momentos en la vida de Frank Ramsey, como que un tìo materno se distanció de él por bolchevique. FrankRamsey hizo una vertiginosa carrera intelectual.

Tuvo una corta y a la vez productiva vida como otros grandes matemáticos de su generación (Otto Schreier 1901-29, Raymond E.A.C. Paley 1907-33, Jacques Herbrand 1908-31). Pero, a diferencia de ellos, Ramsey no fue solo un matemático, sino también un filósofo y un economista. Su obra abarca desde los fundamentos de la matemática, pasando por la economía matemática, la teoría de la decisión y de la probabilidad, hasta la semántica filosófica. Un verdadero polimatemático. 
En 1915 el joven Ramsey entró en el Winchester College y cinco años más tarde obtuvo una beca para realizar sus estudios universitarios de matemáticas, ingresando en elTrinity College en 1920. Pasó cuatro años en el Trinity como estudiante y otros cinco enel King's como profesor, hasta que en 1930 murió poco antes de cumplir 27 años. Lasprimeras tres décadas del siglo XX, el tiempo que le tocó vivir a Ramsey, fueron para Cambridge una suerte de años dorados sólo interrumpida por la Primera Guerra Mundial.

Fueron años de una gran producción científica y filosófica dirigida por personajes de una proliferación intelectual casi sobrehumana. Fueron los años de Rutherford, Eddington, Russell, Moore, Wittgenstein, Braithwaite, Keynes, Strachey,Blunt, Turing, y, por supuesto, Ramsey. De aspecto corpulento, medía casi dos metros y pesaba algo más de cien kilos, y decarácter alegre y tranquilo, Frank Ramsey fue una persona muy querida por su entorno.

En palabras de su amigo Braithwaite, "como persona, no menos que como pensador,Ramsey fue un ornamento para Cambridge. Desde sus días de estudiante se le reconoció como una autoridad en cualquier materia abstracta, y sus aproximaciones directas y candor fueron una inspiración para sus colegas. Su enorme estatura física encajaba bien con el alcance de su intelecto". Keynes lo describió de forma muy parecida en el obituario para The Economic Journal que, a su muerte, le dedicó: "Su corpulenta estructura johnsoniana, su espontánea risa desbordante, la sencillez de sus sentimientos y reacciones, medio alarmante algunas veces y de vez en cuando casi crueles por su espontaneidad y franqueza, su honestidad mental y de corazón, su modestia, y la fácil y sorprendente eficacia de la máquina intelectual que se escondía detrás de sus amplias sienes y de su ancha y sonriente cara, nos han sido arrebatadas en la cima de su excelencia y antes de que su cosecha de trabajo y vida hubiesen podido ser recolectadas".

En su primera publicación pudo verse ya el talento que destilaba Ramsey en sus trabajos. En 1921 J.M.Keynes había publicado su Tratado de la probabilidad. A través de Braithwaite, Ramsey supo de este trabajo y en enero del siguiente año publicó "Mr Keynes on Probability" en The Cambridge Magazine. Keynes vio rápidamente la agudeza de la crítica de Ramsey y reconoció que su teoría había quedado desarticulada en parte por un estudiante de 19 años. A tan temprana edad, según Moore, ya tenía el "excepcional poder de derivar conclusiones desde un complicado conjunto de hechos: podía ver lo que se seguía de ellos tomándolos todos juntos, o, al menos, lo que podría seguirse en casos en los que otros nunca podrían sacar conclusiones". Incluso antes de ingresar en el Trinity College y comenzar los estudios universitarios, cuando todavía vivía en el Magdelene College con su padre, Ramsey ya había deslumbrado a filósofos de la talla de C.K. Odgen e I.A. Richards, al aprender alemán en apenas una semana y de forma autodidáctica con el Análisis de las sensaciones de Mach. Este conocimiento de la lengua germana le serviría para leer, probablemente a finales de ese mismo año, el Logish-Philosophiche Abhandlung de Wittgenstein, la primera versión de lo que terminaría siendo el Tractatus Logico-Philosophicus. A finales de 1921 C.K. Odgen leencomendó la delicada labor de traducir el Tractatus, ello le forzó a pasar el invierno de1921 a 1922 lidiando con el críptico código tractariano.

En 1923 publicó la "Reseña crítica del Tractatus Logico-Philosophicus deL.Wittgenstein", que le había sido requerida desde Mind, su primer trabajo propiamente filosófico. Este trabajo de Wittgenstein, que provocó que en los siguientes diez años, el círculo filosófico de Cambridge intentara digerir al filósofo de origen austriaco, tal como comentó Braithwaite en "Better than the Stars 1 ", produjo una profunda impresión en Ramsey. Ese mismo otoño, Ramsey aceptó la invitación de Wittgenstein para visitarlo, aprovechando que tenía que viajar a Austria para psicoanalizarse. En esa época, Wittgenstein había dejado Cambridge para ir a trabajar de profesor en una pequeña escuela de primaria de una villa austríaca llamada Trattenbach. Pese a que Wittgenstein había terminado su investigación filosófica y por ende había tirado laescalera, tuvo la magnanimidad de volver a dejar subir por ella a Ramsey. Este hecho insólito, difícilmente podría haberse dado si Ramsey, además de tener una favorecida inteligencia, no hubiese tenido el carácter jovial, abierto, tolerante y paciente, por el que también era famoso. Su devastadora risa, como Braithwaite la describió, o ruidosa y pegadiza como prefirió ad- 
jetivarla Mellor, hicieron de Ramsey una de las pocas personas con las que Wittgenstein pudo trabar amistad. Durante 15 días Witggenstein y Ramsey estuvieron comentando cada tarde todos y cada uno de los aforismos del Tractatus. Estos encuentros permitieron a Wittgenstein hacer varias correcciones en la siguiente edición del Tractatus. Por su parte, Ramsey obtuvo algunas ideas para su embrionario proyecto sobre los fundamentos de las matemáticas, en el que queríademostrar, en contra de lo que el Tractatus sentenciaba, que las proposicionesmatemáticas, (o las ecuaciones en opinión de Wittgenstein y Frege), eran tautologías.

Indica Ramsey. "Wittgenstein es profesor en la escuela del pueblo. Es muy pobre, al menos vive muy económicamente. Tiene una habitación diminuta, encalada, que contiene una cama, un lavabo, una mesa pequeña y una silla dura y para eso es todo. Su cena, que compartí anoche, es pan tosco, mantequilla y cacao bastante desagradables. Su horario escolar es de ocho a doce o la una y parece estar libre toda la tarde. Está dispuesto a dedicar cuatro o cinco horas diarias a explicar su libro. He tenido dos días y he leído siete de las ochenta páginas. Él ya ha respondido a mi principal dificultad, sobre la que he estado perplejo durante un año y me rendí desesperado y decidí que no lo había visto. Es terrible cuando dice '¿Está claro?' y yo digo 'No' y él dice 'Maldita sea, es horrible pasar por todo eso de nuevo'." Tras su retorno a Cambridge, en 1924, Ramsey fue elegido Fellow del King's College para dar la asignatura de fundamentos de la matemática. Bajo el patrocinio de Keynes, Ramsey logró este cargo pese a que no fue estudiante del King's. Su carrera académica no terminó aquí, pocos años después llegaría a Director de Estudios Matemáticos del mismo college. Fue Keynes, también, quien finalmente logró persuadir a Wittgenstein para que regresara a Cambridge. Para poder dar clase Wittgenstein necesitaba ser doctor, y Ramsey fue nombrado su supervisor. Más que una supervisión el trabajo que realizaron juntos Ramsey y Wittgenstein fue el de discutir varias veces por semana, durante un buen numero de horas, sobre el trabajo de ambos. Es indudable que Wittgenstein influyó en el trabajo futuro de Ramsey pese a que Ramsey, tras deducir la imposibilidad de reducir las proposiciones matemáticas a tautologías tomó un caminofilosófico bastante alejado al de Wittgenstein. Por otro lado la influencia de Ramsey sobre Wittgenstein tampoco fue menor. El propio Wittgenstein reconoció que fueronRamsey y P. Sraffa quienes más le ayudaron a abandonar las posiciones del Tractatus.

Ese mismo año Ramsey colaboró con Russell en la segunda edición de Principia, pese a que creía que el logicismo de Russell y Whitehead adolecía de errores fundamentales.

En 1925 Ramsey dio una pequeña charla titulada "Eplílogo" para los Apóstoles. LosApóstoles fueron una elitista sociedad de debate fundada en 1820 de la que Ramsey fuemiembro junto a Strachey, Keynes, Russell, Moore y otros grandes personajes delCambridge de principios de siglo XX. A lo largo de los años, prácticamente todas la eminencias intelectuales de Cambridge fueron miembros de este selecto grupo, sin embargo gran parte de su fama a principios de siglo XX se debió más a las tendencias homosexuales y marxistas de muchos de sus miembros que sus privilegiados intelectos.

Ese mismo año, la producción intelectual de Ramsey aumentó sensiblemente. Tras publicar un par de recensiones de la segunda edición de Principia Matemática, salió a laluz su primer artículo propiamente dicho: "Universales", y tras él publicó "Los fundamentos de la matemática". En setiembre de 1925 se casó con Lettice C. Baker con quien tuvo dos hijas. Ramsey conocía a Lettice desde 1921, desde el año en que ingresó en el Trinity, cuando ella estaba en su tercer año de psicología. No hubo boda religiosa, pues en palabras de su propia esposa, Ramsey era un "ateo militante". En 1927 publicó "Lógica matemática" y al año siguiente el célebre artículo "Hechos y proposiciones".

En los cinco años en que mantuvo el cargo de Fellow en el King's publicó once artículos y dejó no publicados, esbozados o inacabados, otros tantos. Casi todos ellos fueron publicados póstumamente en el compendio de Ramsey que hizo Braithwaite en 1931 titulado Los fundamentos de la matemática. La producción científica de Ramsey quedó interrumpida repentinamente a la edad de 25 años. Sufría de una patología llamada ictericia, una enfermedad producida por la acumulación de pigmentos biliares en la sangre. A principios de 1930 tuvo serias dificultades que provocaron que ingresara en el 
Guy's Hospital para que se le practicara una intervención quirúrgica.Frank Plumpton Ramsey murió tras complicaciones en la operación, a las 3 de la mañana de 19 de marzo de 1930.

Su obituario en el Times concluía de la siguiente manera: "Desde sus primeros días de escuela en Cambridge y durante su estancia en el Wichester y en el Trinity, Ramsey fue siempre intelectualmente precoz más allá de su edad y ocupó invariablemente su lugar en términos de igualdad con aquellos que eran varios años mayores que él. Pero junto con su precocidad, combinó una gran simplicidad de mente, de maneras y de carácter. Físicamente, era también de gran tamaño. Sus contemporáneos reconocieron en él a una mente de capacidad y futuro extraordinarios en el territorio entre las matemáticas y la ciencia filosófica y moral. No había nadie entre los jóvenes de Cambridge que pudiera considerarse su igual por potencia y calidad de mente y por brillantez y originalidad de concepción en uno de los temas de estudio más difíciles. Su temprana muerte significa una gravosa pérdida tanto para la ciencia como para sus amigos y colegas."

Ramsey dejó al fallecer un valioso legado en forma de artículos y un profundo vacío en todos sus allegados. Nunca pudo terminar el libro que tenía en mente sobre filosofía de la lógica, del cual solo escribió dos capítulos que luego se publicaron en forma póstuma como un artículo con el nombre: "Truth and Probability", y dejó ciertamente un gran número de brillantes ideas que murieron con él. No sabemos que hubiese sido de él si hubiera sobrevivido a su enfermedad, pero probablemente hubiese asombrado a los filósofos, matemáticos y economistas del siglo XX, una vez más. Quizá se hubiera convertido en el Quine inglés, como fantaseó Ayer, o en el John von Neumann de Cambridge como prefiere imaginar Howson, o quizá hubiese acabado en Bletchley Park con el joven Turing, sin embargo, para desolación de su familia, compañeros y toda la comunidad intelectual ese día nunca llegó. "Better than the Stars" es el título de un reportaje radiofónico acerca de la vida y obra de F.Ramsey emitido por la BBC en 1978.

\subsection{Obra científica de Ramsey}

Ramsey se destacó entre otras en: filosofía analítica, economía y fundamentos de la matemática. En economía se le recuerda por los 3 artículos publicados -uno de ellos postmortem. Voy a presentar ahora tres traducciones de recensiones de Remsey:

El Sr. Keynes sobre Probabilidad (1922) (Aquí Ramsey con 19 años realiza una crítica a la teoría de probabilidades de Keynes, un reconocido científico). Traducción María José Frápolli

El Sr. Keynes toma la probabilidad o las relaciones de probabilidad como indefinibles, y dice que si q tiene con $p$ la relación de probabilidad de grado $a$, entonces el conocimiento de $p$ justifica la creencia racional de grado a en q. Tenemos entonces numerosas relaciones de probabilidad; estas se supone comúnmente que son todas numéricas, esto es, correlacionadas con los números reales desde 0 a 1 de tal modo que las reglas normales del cálculo de probabilidades se dan, por ejemplo, que el producto de los números correlacionados con dos probabilidades es igual al número correlacionado con el producto (en el sentido del Sr. Keynes) de las dos probabilidades. El Sr. Keynes niega esto; supone no sólo que no todas las probabilidades son numéricas, sino también que es posible tener dos probabilidades que no sean iguales y tales que ninguna sea mayor que la otra. Esta concepción se basa en la dificultad en tantos casos de decir con alguna confianza cuál de dos probabilidades es la mayor, o de asignarles cualesquiera medidas numéricas. Pero parece que la fuerza de esta objeción a la concepción común se exagera por parte del Sr. Keynes por dos razones.

Primero, cree que entre cualesquiera dos proposiciones no autocontradictorias se da una relación de probabilidad (Axioma I), por ejemplo entre "mi alfombra es azul" y "Napoleón fue un 
gran general"; se ve fácilmente que lleva a contradicciones asignar la probabilidad $1 / 2$ a tales casos, y el Sr. Keynes concluirá que la probabilidad no es numérica. Pero parecería que en tales casos no hay ninguna probabilidad; para que entre dos proposiciones se de una relación lógica distinta de una función de verdad, debe haber alguna conexión entre ellas. Si esto es así, no hay ninguna probabilidad como la probabilidad de "mi alfombra es azul" dado sólo que "Napoleón fue un gran general", no existe por tanto el problema de asignarle un valor numérico.

En segundo lugar, es seguramente obvio que las probabilidades pueden ser numéricas o comparables sin que seamos capaces de asignarles sus valores numéricos o compararlas, debido a la imperfección de nuestra intuición lógica. Así una probabilidad podría, como el Sr. Keynes admite, ser desconocida para nosotros por falta de capacidad para argumentar a partir de una evidencia dada. Pero dice "No debemos permitir que esta admisión nos lleve demasiado lejos. La probabilidad es relativa en un sentido a los principios de la razón humana. El grado de probabilidad que es racional para nosotros mantener no supone una intuición lógica perfecta, y es en parte relativo a las proposiciones secundarias que de hecho conocemos; ... Si no adoptamos esta visión de la probabilidad, si no la limitamos de esta manera y la hacemos, hasta este punto, relativa a los poderes humanos, estamos completamente a la deriva en lo desconocido; porque ni siquiera podemos conocer qué grado de probabilidad estaría justificado por la percepción de las relaciones lógicas que somos, y debemos ser siempre, incapaces de comprender."

Pero nos preocupa la relación que realmente se da entre dos proposiciones; a la facultad de percibir esta relación, con precisión o no, la llamamos intuición perfecta o imperfecta. El Sr. Keynes argumenta que debido a la posibilidad de que nuestra intuición pueda estar completamente equivocada no deberíamos hablar de relaciones que realmente se dan, sino de relaciones de las que tenemos razones para suponer que se dan. Entonces, piensa, podríamos hablar sin temer a factores desconocidos. No parece haber, sin embargo, ninguna buena razón para confinar este argumento a la probabilidad. En todo, podría defenderse, debido a la posibilidad de que haya evidencia a la que no tenemos acceso, sólo estamos justificados a decir no ya " $p$ " sino "tenemos razones para suponer $p$ ". La conclusión lógica en esta concepción es que no estamos justificados a decir nada en absoluto; porque nuestra evidencia acerca de la razón humana podría también ser fragmentaria. No podemos, por tanto, decir razonablemente "tenemos razones para suponer que la probabilidad es a", sino sólo "tenemos razones para suponer que tenemos razones para suponer que la probabilidad es a", y así sucesivamente ad Infinitum -- en la línea del celebrado argumento de la Ética del Dr. Moore.

El Sr. Keynes es como un supervisor, que temiendo que sus estimaciones de las alturas de las montañas pudieran ser erróneas, decide que si hablara acerca de alturas reales estaría a la deriva en lo desconocido; así diría que las alturas eran relativas a los instrumentos del supervisor, y cuando llegara a una montaña oculta en la niebla le asignaría una altura no numérica porque no podría ver si era más alta o más baja que otras.

Después de tratar con la medición de las probabilidades, el Sr. Keynes procede a considerar el Principio de Indiferencia, que muestra que lleva, si se afirma en su forma habitual, a diversas contradicciones. Él propone remediar esto enunciando condiciones precisas para la validez del principio. No parece, sin embargo, haber realizado esto con éxito. Al final de la pág. 62 dice: Supongamos que un punto cae en una línea de longitud $\mathrm{ml}$, podemos escribir la alternativa "el intervalo de longitud $l$ en el que el punto está es el $x$-ésimo ... de izquierda a derecha $\varphi(x)$; y el Principio de Indiferencia puede entonces aplicarse con seguridad a las $m$ alternativas $\varphi(1)$, $\varphi(2) \ldots(\mathrm{m})$ " y claramente este caso cae bajo sus condiciones; y así lo hace el caso análogo en que sabemos que la densidad de una sustancia cae entre 1 y 3; podemos tomar entonces "intervalo de longitud 1 en el que cae la densidad es el $x$-ésimo de izquierda a derecha" $\varphi(x)$ y aplicar el Principio a $\varphi(1), \varphi(2)$, concluyendo que es igualmente probable que la densidad caiga en los 
intervalos 1 - 2 y 2 - 3; si ahora aplicamos también este argumento al volumen específico que consideramos que está entre 1 y $1 / 3$, puesto que la densidad cae entre 1 y 3 , encontramos que, sobre los mismos datos, es igualmente probable que el volumen específico caiga en los intervalos $1-2 / 3,2 / 3-1 / 3$ y por tanto la densidad en los intervalos $1-2 / 3,3 / 2-3$, lo que contradice el resultado previamente obtenido. Esta contradicción la señala el Sr. Keynes, pág. 45, pero parece no haber notado que escapa a sus defensas.

La verdadera solución a la dificultad parece depender de la noción del Sr. Johnson de "Lo Determinable". El Principio de Indiferencia podría formularse como sigue: relativamente a la evidencia, sobre la que es cierto que un sujeto dado tiene uno u otro de entre un número finito de determinados absolutos bajo el mismo determinable, las probabilidades de que el sujeto tenga cada uno de estos determinados absolutos son iguales, siempre y cuando la evidencia sea simétrica en relación a las distintas alternativas.

El principio, así cualificado, puede aplicarse a dados, monedas y cartas, pero no a casos como la posición de un punto en una línea, en los que el número de determinados absolutos posibles (por ejemplo, los puntos de la línea) es infinito. Parece que no puede darse ningún principio para casos de este segundo tipo que no llevarían a contradicción como los del volumen o la densidad. La conclusión natural es que en tales casos no hay ninguna probabilidad; esto es, que no hay ninguna relación lógica entre las premisas y la conclusión.

En la Parte II, el Sr. Keynes da una deducción simbólica de las fórmulas del cálculo de probabilidades a partir de definiciones y axiomas; ésta tiene un pequeño fallo. El Sr. Keynes oculta dos importantes axiomas como definiciones; al definir la suma de $a b / h, a \sim b / h$ como $a / h$ y el producto de $a / b h, b / h$ como $a b / h$, oculta las asunciones de que la suma y el producto así definidos son siempre únicos, esto es, que si $a b / h=c d / k,(=P), a \sim b / h=c \sim d / k,(=P Q) ; y$ que si $\mathrm{a} / \mathrm{bh}=\mathrm{c} / \mathrm{dk},(=\mathrm{P}), \mathrm{b} / \mathrm{h}=\mathrm{d} / \mathrm{k},(=\mathrm{Q})$ entonces $\mathrm{ab} / \mathrm{h}=\mathrm{cd} / \mathrm{k},(=\mathrm{PQ})$.

El tratamiento que el Sr. Keynes da a la inducción parece estar viciado por el hecho de que sólo considera el Método del Acuerdo, ignorando completamente los otros cuatro métodos de Mill, incluido, por ejemplo, el Método de la Diferencia, inferir $g(\varphi, f)$, no a partir de muchos casos, por otra parte tan variados como sea posible que coincidan en tener $\varphi f$, sino de conjuntos de dos casos, análogos en los demás aspectos, uno que tenga $\varphi$ f y en otro no $\varphi$, no f.

El Sr. Keynes concluye que la inducción sólo es racional si hay una probabilidad finita a priori a favor de lo que él llama la Hipótesis de la Variedad Independiente Limitada; esto es, que todas las propiedades surjan a partir de un número finito de propiedades generadoras. Si hay que tomar esto literalmente, esto es, "propiedad" interpretada en el sentido amplio = función proposicional de una variable, es claramente equivalente a la hipótesis de que las clases de cosas del tipo considerado son finitas en número, puesto que propiedades equivalentes definen la misma clase y por la hipótesis cualquier propiedad es equivalente a una de entre un número finito de propiedades (esto es, las propiedades generadoras y las negaciones, conjunciones y disyunciones de ellas). Y esta hipótesis de que las clases de cosas son finitas en número es, a su vez, equivalente a la hipótesis de que las cosas son finitas en número, puesto que, si $n$ es el número de cosas, $2 n$ es el número de clases de cosas; de modo que la Hipótesis de la Variedad Limitada es simplemente equivalente a la contradictoria del Axioma de Infinitud.

Finalmente podemos señalar que la definición del Sr. Keynes de "azar" sugiere que podría estar equivocado en su concepción fundamental de la probabilidad. Porque en ella ocurre la probabilidad $\varphi(x) / S(x) h$; y se considera si es igual a $\varphi(x) / S(x) \cdot h \cdot x=a=\varphi(a) / S(a) \cdot h$. 
Ahora bien en $\varphi(x) / S(x) \cdot h$, $x$ es una variable. $\varphi(x), S(x)$ no son proposiciones en absoluto sino funciones proposicionales. Tenemos, por tanto, un nuevo tipo de probabilidad, una relación entre dos funciones proposicionales, $\varphi(x), S(x)$ y una proposición $h$; un tipo que no puede reducirse al tipo común (una relación entre dos proposiciones). Pero la reducción conversa (excepto en la concepción de la identidad del Sr. Wittgenstein) siempre es posible, por ejemplo $\varphi(a) / S(a) \cdot h=\varphi(x) / S(x) \cdot x=a \cdot h$. Tenemos, por tanto, dos posibilidades; o bien hay dos tipos de relaciones de probabilidad, relaciones diádicas entre proposiciones, y relaciones triádicas entre dos funciones proposicionales y una proposición; o bien todas las relaciones de probabilidad son del último tipo, más complicado.

Fragmentos de la recensión Crítica del Tractatus de Wittgenstein (1923) Traducción: Francesc Camós.

Este es un libro de lo más importante que contiene originales ideas acerca de una amplia gama de temas, conformando un sistema coherente, que sea o no, como el autor afirma, en lo esencial la solución final de los problemas de los que trata, es de un extraordinario interés y merece la atención de todos los filósofos. Incluso si el sistema fuera en conjunto incorrecto el libro contiene un amplio número de profundas obiter dicta y críticas a otras teorías. Sin embargo, el libro es difícil de entender, a pesar del hecho de que está impreso con el texto alemán y la traducción inglesa en páginas encontradas. El Sr. Wittgenstein no escribe prosa consecutiva, sino oraciones cortas numeradas de tal modo que muestran el énfasis puesto sobre ellas en su exposición. Esto le da a su trabajo un atractivo aroma epigramático, y quizá le confiere más exactitud en el detalle, en el sentido de que cada oración debe haber recibido consideración por separado; pero esto parece haberle impedido dar explicaciones adecuadas de muchos de sus términos técnicos y teorías, quizá porque las explicaciones requieren sacrificar cierto rigor.

Esta deficiencia está subsanada en parte en la introducción del Sr. Russell; pero es posible que él no sea una guía infalible para lo que el Sr. Wittgenstein quiere decir. "A fin de entender el libro del Sr. Wittgenstein," dice el Sr. Russell, "es necesario darse cuenta de cuál es el problema en el que está interesado. En la parte de su teoría en la que se ocupa del Simbolismo le interesan las condiciones que habrían de satisfacerse por un lenguaje lógicamente perfecto". Esta parece ser una generalización muy dudosa; hay, en efecto, pasajes en los que el Sr. Wittgenstein está explícitamente interesado no en cualquier lenguaje sino en los lógicamente perfectos, e.g. la discusión sobre "sintaxis lógica" en 3.325 ss; pero en general parece mantener que sus doctrinas se aplican a lenguajes corrientes a pesar de las apariencias de lo contrario (ver especialmente 4.002 y ss.). Obviamente este es un punto importante, porque esta aplicación tan amplia aumenta enormemente el interés y disminuye la plausibilidad de cualquier tesis como la que el Sr. Russell declara que es quizá la más fundamental de la teoría del Sr. Wittgenstein; que "a fin de que una cierta oración pueda aseverar un cierto hecho debe haber algo en común entre la estructura de la oración y la estructura del hecho, cualquiera que sea la manera en la que el lenguaje pueda construirse". Esta doctrina parece depender de las complicadas nociones de una "figura" y su "forma de representación", que ahora intentaré explicar y criticar.

Una figura es un hecho, el hecho de que sus elementos se combinen unos con otros de un modo determinado. Estos elementos se coordinan con ciertos objetos (los constituyentes del hecho del cual la figura es una figura). Estas coordinaciones constituyen la relación de representación que hace a la figura una figura. Esta relación de representación "pertenece a la figura" (2.1513); esto, creo, significa que siempre que hablamos de una figura tenemos en mente alguna relación de representación en virtud de la cual eso es una figura. Bajo estas circunstancias decimos que la figura representa que los objetos están combinados de tal modo unos con otros como están los elementos de la figura, y éste es el sentido de la figura. Creo que esto debe ser tomado como la definición de "representa" y de "sentido"; es decir, que cuando decimos que una figura repre- 
senta que ciertos objetos están combinados de un cierta modo, queremos decir meramente que los elementos de la figura están combinados de este modo, y están coordinados con los objetos por la relación de representación que pertenece a la figura. (que esto es una definición se sigue, creo, de 5.542.)

Puede arrojarse luz sobre la "forma de representación" con las siguientes observaciones hechas anteriormente en el libro sobre la estructura y la forma de los 30 hechos. "El modo en que los objetos encajan juntos en el hecho atómico es la estructura del hecho atómico. La forma es la posibilidad de la estructura. La estructura del hecho consiste en las estructuras de los hechos atómicos". $(2.032,2.033,2.034)$. El único interés que puedo ver en la distinción entre estructura y forma es que la inserción de "posibilidad" puede incluir el caso en el que el supuesto hecho cuya forma estamos considerando no sea un hecho, de modo que podemos hablar de la forma del hecho $\mathrm{aRb}$, sea o no $\mathrm{aRb}$ verdadero, en el caso de que sea lógicamente posible. Es lamentable que las definiciones anteriores no aclaren si dos hechos pueden tener en algún caso la misma estructura o la misma forma; Parece como si dos hechos atómicos pudieran tener la misma estructura porque los objetos encajen de la misma manera en cada uno de ellos. Pero parece por observaciones posteriores en el libro que la estructura del hecho no es meramente la manera en la que los objetos encajan juntos sino que depende también de qué objetos son, de tal modo que dos hechos diferentes nunca tienen la misma estructura...

Pasemos ahora a la justificación del Sr. Wittgenstein de la filosofía. "El objeto de la filosofía", dice, "es la clarificación lógica de los pensamientos. La filosofía no es una teoría sino una actividad. Un trabajo filosófico consiste esencialmente en elucidaciones. El resultado de la filosofía no es un número de "proposiciones filosóficas", sino clarificar proposiciones. La filosofía debería clarificar y delimitar nítidamente los pensamientos que de otra forma son, por así decirlo, opacos y borrosos" (4.112). Me parece que no podemos estar satisfechos con este enfoque sin alguna ulterior explicación de "claridad", e intentaré dar una explicación en armonía con el sistema del Sr. Wittgenstein. Creo que una oración escrita es "clara" en la medida en que tiene propiedades visibles correlacionadas con o "mostrando" las propiedades internas de su sentido. De acuerdo con el Sr. Wittgenstein las últimas siempre se muestran a sí mismas en propiedades internas de la proposición; pero debido a la ambigüedad ejemplar-tipo de "proposición" no está inmediatamente claro lo que esto significa. Las propiedades de una proposición tiene que significar, creo, propiedades de todos sus ejemplares; pero las propiedades internas de una proposición son aquellas propiedades de los ejemplares que son, por así decirlo, internas no a los ejemplares sino al tipo; esto es, aquellas que uno de los ejemplares debe tener si tiene que ser un ejemplar de este tipo, no aquellas que es impensable que no tuviera de algún modo.

Tenemos que recordar que no hay necesidad de que una oración tenga el sentido que de hecho tiene. Así que si una oración dice fa, no es un propiedad interna de la oración que haya algo en ella de alguna forma conectado con a; pero esto es una propiedad interna de la proposición, porque la oración no podría pertenecer en caso contrario a esta proposición tipo, i.e. tener este sentido. Así vemos que las propiedades internas de una proposición que muestran aquellas de su sentido no son en general, visibles, sino [propiedades] complejas que implican la noción de significado. Pero en un lenguaje perfecto en el que cada cosa tuviera su propio nombre único, que en el sentido de una oración un cierto objeto apareciera, se mostraría también visiblemente por la aparición en la oración del nombre de ese objeto; y esto es lo que se esperaría que pasara con respecto a todas las propiedades internas de los sentidos; que un sentido, por ejemplo, está contenido en otro (i.e. una proposición se sigue de otra) podría siempre aparecer visiblemente en las oraciones que los expresaran. (Esto está prácticamente logrado en la notación V del Sr. Wittgenstein.) De esta manera en un lenguaje perfecto todas las oraciones o pensamientos serían perfectamente claros. Para dar una definición general de "claro" debemos reemplazar "propiedad visible de la oración" por "propiedad interna del signo proposicional", que interpretamos análogamente a "propiedad interna de la proposición" como una propiedad que un ejemplar 
debe que tener si tiene que ser este signo, que, si el ejemplar está escrito, es lo mismo que una propiedad visible. Decimos entonces que un signo proposicional está claro en la medida en que las propiedades internas de su sentido se muestran no solo por las propiedades internas de la proposición sino también por las propiedades internas del signo proposicional.

\section{Recensión sobre Lógica Matemática (1926). Traducción María José Frápolli}

Se me ha pedido que hable acerca de los desarrollos en lógica matemática desde la publicación de Principia Mathemática, y creo que sería de lo más interesante si en lugar de describir las diversas mejoras definidas en detalle, discutiera a grandes rasgos el trabajo que se ha hecho sobre líneas completamente distintas y que reclama sustituir completamente a la posición considerada por Whitehead y Russell acerca de la naturaleza de las matemáticas y sus fundamentos lógicos.

Comenzaré recordando cuál es la posición de Whitehead y Russell: es que las matemáticas son parte de la lógica formal, que todas las ideas de las matemáticas puras pueden definirse en términos que no son distintivamente matemáticos sino que están implicados en el complicado pensamiento de cualquier descripción y que todas las proposiciones de las matemáticas pueden deducirse de proposiciones de la lógica formal, tales como que si p es verdadero, entonces o $\mathrm{p}$ o q es verdadero. Esta concepción me parece plausible en sí misma, porque tan pronto como la lógica se ha desarrollado más allá de su viejo núcleo silogístico, podemos esperar tener junto con las formas "todos los hombres son mortales", "algunos hombres son mortales", las formas numéricas "dos hombres son mortales" y "tres hombres son mortales", y el número tendrá que incluirse en la lógica formal.

Frege fue el primero en mantener que las matemáticas eran parte de la lógica y en construir una teoría detallada sobre esta base. Pero cayó víctima de las famosas contradicciones de la teoría de los agregados, y pareció que podían deducirse consecuencias contradictorias de sus proposiciones primitivas. Whitehead y Russell escaparon a este destino introduciendo su Teoría de los Tipos, de la cual es imposible aquí dar un tratamiento adecuado. Pero una de sus implicaciones debe explicarse si queremos que sean inteligibles desarrollos posteriores.

Supongamos que tenemos un conjunto dado de características como todas las características de una cierta clase, digamos A, entonces podemos preguntar acerca de si algo tiene una característica de la clase A. Si la tiene, esto será otra característica de eso, y se plantea la cuestión de si esta característica, la característica de tener una característica del tipo A, puede ser ella misma del tipo A, viendo que presupone la totalidad de tales características. La Teoría de los Tipos mantenía que no podía, y que solo podríamos escapar a la contradicción diciendo que era una característica de orden superior, y que no podría incluirse en un enunciado acerca de todas las características de orden inferior. Y más generalmente que cualquier enunciado acerca de todas las características debe considerarse como si significara todas las de un cierto orden. Esto pareció plausible en sí mismo, y también el único modo de evitar ciertas contradicciones que aparecían al confundir estos órdenes de características. Whitehead y Russell mantuvieron también que los enunciados acerca de clases o agregados tienen que considerarse como si realmente fueran acerca de las características que definen las clases (siempre siendo una clase dada la clase de las cosas que poseen un cierto carácter), de tal modo que cualquier enunciado acerca de todas las clases será realmente acerca de todas las características, y estará sometido a las mismas dificultades en relación al orden de estas características.

Esta teoría fácilmente nos permite evitar las contradicciones de la Teoría de los Agregados, pero tiene también la desafortunada consecuencia de invalidar un tipo común e importante de argumento matemático, la clase de argumento mediante el cual en última instancia establecemos la existencia de un límite superior de un conjunto, o la existencia de un límite de una sucesión monotónica acotada. Es usual deducir estas proposiciones a partir del principio del corte de 
Dedekind, que si los números reales se dividen completamente en una clase superior y en una clase inferior, debe haber un número que los divida que será el menor de la clase superior o el mayor de la inferior. Esto a su vez se prueba considerando a los números reales como cortes de racionales; cortes de racionales son un tipo particular de clases de racionales, y así un enunciado acerca de números reales será un enunciado acerca de un tipo de clase de racionales, que es acerca de un tipo de características de racionales y las características en cuestión tendrán que limitarse a ser de un cierto orden.

Esto significa que el análisis tal como se entiende comúnmente está completamente fundado sobre un tipo de argumento falaz, que cuando se aplica en otros campos lleva a resultados autocontradictorios.

Whitehead y Russell tratan de evitar esta desafortunada consecuencia de la Teoría de los Tipos introduciendo el Axioma de Reducibilidad, que afirma que para toda característica de orden superior hay una característica equivalente del orden más bajo equivalente en el sentido de que todo lo que tiene la una lo tiene la otra, de tal modo que definen la misma clase. El límite superior, que vimos que era una clase de racionales definida mediante una característica de orden superior sería entonces definido también mediante una característica equivalente de orden inferior, y sería un número real. Desafortunadamente este axioma no es ciertamente autoevidente, y no hay razón alguna para suponer que es verdadero. Si fuera verdadero esto sería, por así decirlo, un feliz accidente, y no sería una verdad lógica como las otras proposiciones primitivas. En la Segunda Edición de Principia Mathemática, de la cual el primer volumen se publicó el año pasado, el Sr. Russell ha mostrado cómo la inducción matemática, para la que el Axioma de Reducibilidad parece también exigirse, puede establecerse sin él, pero no mantiene ninguna esperanza de un éxito similar con la Teoría de los Números Reales, para la que el ingenioso método usado para los números enteros no está disponible. La materia se ha dejado así en una condición profundamente insatisfactoria.

Esto fue apuntado por Weyl, quien publicó en 1918 un librito llamado Das Kontinuum, en el que rechazaba el Axioma de Reducibilidad y aceptaba la consecuencia de que el análisis común estaba equivocado. Mostró, sin embargo, que varios teoremas, como el Principio General de Convergencia de Cauchy, todavía podían probarse. Desde entonces Weyl ha cambiado su posición y se ha convertido en un seguidor de Brouwer, el líder de lo que se conoce como la escuela intuicionista, cuya doctrina principal es la negación de la Ley del Tercero Excluido, que toda proposición es o verdadera o falsa. Se la niega aparentemente porque se piensa que es imposible conocer tal cosa a priori, e igualmente imposible conocerla por experiencia, porque si no sabemos que es o verdadera o falsa no podemos verificar que es o verdadera o falsa. Brouwer rehusaría conceder que o estaba lloviendo o no estaba lloviendo, a menos que hubiera mirado a ver. Aunque es ciertamente difícil dar una explicación científica de nuestro conocimiento de las leyes de la lógica, no me puedo convencer de que no sé con certeza que la Ley del Tercero Excluido es verdadera; por supuesto que no puede probarse, aunque Aristóteles dio el siguiente argumento ingenioso en su favor. Si una proposición no es ni verdadera ni falsa, llamémosla dudosa; pero entonces si la Ley del Tercero Excluido es falsa, no tiene que ser ni dudosa ni no dudosa, así no tenemos simplemente tres posibilidades sino cuatro, que es verdadera, que es falsa, que es dudosa, y que no es ni verdadera, ni falsa, ni dudosa. Y así sucesivamente ad infinitum.

\section{Conocimiento (1929). Traducción Francesc Camós Abril.}

Siempre he dicho que una creencia era conocimiento si era (i) verdadera, (ii) cierta, (iii) obtenida mediante un proceso fiable. Pero la palabra "proceso" es muy insatisfactoria; podemos llamar a la inferencia un proceso, pero incluso entonces no fiable parecen hacer referencia sólo a un método falaz, no a premisas falsas como se supone que [debería] hacer. ¿Podemos decir que 
un recuerdo se obtiene mediante un proceso fiable? Creo que quizás podemos si nos referimos al proceso causal que conecta lo que ocurre con el hecho de que lo recuerde. Entonces podríamos decir, una creencia obtenida mediante un proceso fiable tiene que estar causada por lo que en cierta forma no son creencias o con acompañamientos que pueden ser más o menos fiables para dar creencias verdaderas, y si en esta cadena causal concurren otras creencias intermedias, estas tienen que ser todas verdaderas.

Por ejemplo “¿Es conocimiento la telepatía?" puede querer decir: (a) Suponiendo que haya un proceso tal, ¿podría ser fiable para crear creencias verdaderas en el telépata (dentro de algunos límites, p. e cuando lo que se cree es acerca de los pensamientos del telépata o (b) Suponiendo que seamos agnósticos ¿el sentimiento de ser conectado telepáticamente garantiza la verdad? Lo mismo digo para la intuición femenina, impresiones del carácter, etc. Quizá no deberíamos decir (iii) obtenida mediante un proceso fiable, sino (iii) formada por un camino fiable. Decimos "lo sé", sin embargo, siempre que estamos seguros, sin reflexionar acerca de la fiabilidad. Pero si meditáramos entonces deberíamos mantenernos seguros si, y solo si, creyéramos que el camino es fiable. (Supongamos que lo sabemos; si no, sería lo mismo tomarlo meramente como una descripción, por ejemplo, Dios lo puso en mi mente: un proceso supuestamente fiable.) Porque pensar que el camino es fiable es simplemente formular en un [enunciado] hipotético variable el hábito de seguir el camino.

Otra cosa más. Russell dice en sus Problems of Philosophy que no hay duda de que a veces estamos equivocados, de modo que todo nuestro conocimiento está infectado con algún grado de duda. Moore solía negar esto, diciendo que por supuesto esto era auto-contradictorio, que es mera pedantería e ignorancia del tipo de conocimiento propuesto. Pero substancialmente el asunto es éste: no podemos decir sin auto- contradicción $p$ y q y $r$ y ... y una de las $p, q, r$... es falsa. (N.B. -Sabemos lo que sabemos, de otra forma no habría contradicción). Pero podemos estar prácticamente seguros de que una es falsa y sin embargo casi seguros de cada una de ellas; pero $p, q, r$ están entonces infectadas con la duda. Pero Moore está en lo cierto al decir que no están todas infectadas necesariamente; pero si exceptuamos algunas, probablemente nos aclararemos bastante acerca de que una de las exceptuadas está probablemente equivocada, y así sucesivamente.

Los dos artículos de economía siguen generando conocimientos.

El teorema de Ramsey es esencial en lógica y teoría de grafos, sus aplicaciones son múltiples.

La teoría de tipos de la cual Ramsey es uno de los grandes desarrolladores, es una herramienta fundamental en campos tales como la demostración automática de teoremas.

\section{Bibliografía}

[1] Arrow, K (1980). “Review: Foundations: Essays in Philosophy, Logic, Mathematics and Economics" (PDF). Journal of Political Economy. 88 (3): 636-638. https : //doi . org/10. $1086 / 260894$.

[2] Bernstein, B. A. (1932). “Review: The Foundations of Mathematics, and other Essays by Frank Plumpton Ramsey, edited by R. B. Braithwaite, preface by G. E. Moore" (PDF). Bull. Amer. Math. Soc. 38 (9): 611-612. https://doi.org/10.1090/s0002-9904- 1932 05470 - 2 
[3] Duarte, Pedro G (2009a). "Frank P. Ramsey: A Cambridge Economist". History of Political Economy. 41 (3): 445-470. https://doi.org/10.1215/00182702-2009-035. S2CID144949987.

[4] Duarte, Pedro G. (2009b). "Frank Ramsey's Notes on Saving and Taxation". History of Political Economy. 41 (3): 471-489. https: //doi.org/10. 1215/00182702-2009- 048.

[5] Forrester, John (2004). "Freud in Cambridge" (PDF). Critical Quarterly. 46 (2): 1-26. https://doi.org/10.1111/j.0011-1562.2004.t01-1-00560.x. Archived from the original (PDF) on 22 May 2011. Retrieved 26 July 2010.

[6] Galavotti, M. C. (Ed.) (2006), Cambridge and Vienna: Frank P. Ramsey and the Vienna Circle, Dordrecht, The Netherlands: Springer.

[7] Grattan-Guinness, Ivor (2000), The Search for Mathematical Roots 1870-1940, Princeton University Press, Princeton, NJ.

[8] Keynes, John Maynard (1933), “F. P. Ramsey”, in Essays in Biography, New York, NY.

[9] Mellor, D.H. (1995). “Cambridge Philosophers I: F. P. Ramsey". Philosophy. 70 (272): 243-262. https://doi.org/10.1017/s0031819100065396.

[10] Newbery, D. "Ramsey model”. The New Palgrave: A Dictionary of Economics. 4: 46-48.

[11] Newman, P (1987). "Ramsey, Frank Plumpton". The New Palgrave: A Dictionary of Economics. 4: 41-46.

[12] Ramsey, F.P. (1927). "Facts and Propositions" (PDF). Aristotelian Society Supplementary. 7: 153-170. https://doi.org/10.1093/aristoteliansupp/7.1.153.

[13] Ramsey, F.P. (1928). "A Mathematical Theory of Saving". Economic Journal. 38 (152): 543-559. https://doi .org/10.2307/2224098. JSTOR2224098.

[14] Ramsey, F.P. (1927). "A Contribution to the Theory of Taxation". Economic Journal. 37 (145): 47-61. https://doi . org/10.2307/2222721. JSTOR 2222721.

[15] Ramsey, F.P. (1929). "On a Problem in Formal Logic" (PDF). Proc. London Math. Soc. 30: 264-286. https://doi.org/10.1112/plms/s2-30.1.264.

[16] Ramsey, F.P. (1931), The Foundations of Mathematics, and other Essays, (ed.) R. B. Braithwaite Ramsey, F.P. (1978) Foundations - Essays in Philosophy, Logic, Mathematics and Economics, (ed.) D.H. Mellor, Humanities Press, LCCN 77-26864 F. P. Ramsey. Obra Filosófica Completa. M.J. Frápolli (2005), Edición, introducción y traducción. Granada, Comares

[17] Rescher, Nicholas and Ulrich Majer (eds.) (1991). F. P. Ramsey: On Truth, Dordrecht, Kluwer

[18] Sahlin, N.-E. (1990), The Philosophy of F. P. Ramsey, Cambridge University Press, Cambridge

[19] Sahlin, N.-E. (1996), "He is no good for my work": On the philosophical relations between Ramsey and Wittgenstein, in Knowledge and Inquiry: Essays on Jaakko Hintikkas Epistemology and Philosophy of Science, ed by M. Sintonen, Poznan Studies in the Philosophy of Sciences and the Humanities, Amsterdam, 61-84

[20] Sahlin, N.-E. (2005), Ramsey's Ontology, a special issue of Metaphysica, No. 3

[21] Samuelson, P (1970). “What Makes for a Beautiful Problem in Science?". Journal of Political Economy. 78 (6): 1372-1377. https://doi.org/10. 1086/259716. 